\title{
Measured scaling properties of inhomogeneous turbulent flows
}

\author{
P. Tong \\ Department of Physics, Oklahoma State University, Stillwater, Oklahoma 74078 \\ W. I. Goldburg \\ Department of Physics and Astronomy, University of Pittsburgh, Pittsburgh, Pennsylvania 15260 \\ J. S. Huang \\ Exxon Research and Engineering Company, Annandale, New Jersey 08801
}

(Received 9 September 1991)

\begin{abstract}
A turbulent flow lacking homogeneity and isotropy has been studied using the technique of photoncorrelation homodyne spectroscopy to measure velocity differences in different directions and at various length scales. The measured intensity correlation function $G(q t, L)$ is found to be of the scaling form $G(q t u(L))$ with $u(L) \sim L^{\xi}$, where $u(L)$ is the characteristic turbulent velocity at the length scale $L$ and $q$ is the scattering vector. The exponent $\xi$ varies with scattering geometries and with flow cells. The scaling behavior of $G(q t, L)$ is found to be independent of spatial positions and orientations. A scaling $G(q t u(L))$ indicates that the velocity-density function $P(V, R)$ has a scaling form $Q(V / u(R)) / u(R)$. The experiment suggests that the scaling argument can still be used to describe anisotropic and inhomogeneous turbulent flows, and that the exact functional form of $P(V, R)$ (which is of scaling form) may vary with spatial positions and orientations in turbulent flows, reflecting the inhomogeneity and anisotropy of the turbulence.
\end{abstract}

PACS number(s): 47.55. $-\mathrm{t}, 47.25 .-\mathrm{c}, 42.25 .-\mathrm{p}, 05.40 .+\mathrm{j}$

\section{INTRODUCTION}

The Kolmogorov theory [1] for homogeneous and isotropic turbulence has played an important role in the study of the statistical mechanics of turbulence. According to the theory, the kinetic energy is fed into the system at the largest scale $l_{0}$, which is determined by the boundary of the system. The energy is continuously transferred from eddies of size $R<l_{0}$ to eddies of smaller size, until it dissipates when the size of eddies becomes comparable to the viscous dissipation length $l_{d}$. In the inertial range $l_{d}<R<l_{0}$, the energy cascades at a rate $\epsilon$ without dissipation. As a consequence of the argument, the velocity difference $V(R, t)$ between two points in a turbulent fluid separated by a distance $R$ is expected to be scale invariant in that the statistical properties of $V(R, t)$ over varying length scales $R$ become identical under an appropriate scaling of velocities [2-4]. This greatly simplifies the statistical description of turbulence. The velocity moments $\left\langle V(R, t)^{n}\right\rangle$ become a simple power of the scaling velocity $u(R)$ associated with eddies of size $R$, and a simple dimensional argument gives $[1,3] u(R) \simeq(\epsilon R)^{1 / 3}$. It is easy to show that the above scaling result follows if the probability-density function $P(V, R)$ of $V(R, t)$ is a homogeneous function $[5,6]$ :

$$
P(V, R)=Q(V / u(R)) / u(R) .
$$

An interesting question one might ask is, what happens for most "real" turbulent flows which are neither homogeneous nor isotropic? In a recent paper Knight and Sirovich [7] have shown that the above scaling arguments for homogeneous and isotropic turbulence can be extend- ed to certain turbulent flows lacking homogeneity and isotropy. In this paper we report a light-scattering study of such a turbulent flow, from which the functional form of $P(V, R)$ can be inferred. The experiment suggests that the velocity-density function $P(V, R)$ in our inhomogeneous and anisotropic turbulent flow has the scaling form shown in Eq. (1). This agrees with the scaling arguments by Knight and Sirovich. Furthermore, it is found that the exact functional form of $P(V, R)$ (which is of scaling form) varies with spatial positions and orientations in the turbulent flow, reflecting the inhomogeneity and anisotropy of the turbulence.

It has been shown $[6,8,9]$ that the density function $P(V, R)$ is accessible by the technique of photoncorrelation homodyne spectroscopy (HS) [10]. The HS technique differs from the standard one of laser Doppler velocimetry (LDV), [11,12] in that LDV measures the local velocity $\mathbf{v}(\mathbf{r}(\mathrm{t}))$ whereas photon-correlation spectroscopy senses the instantaneous velocity difference $\mathbf{V}(\mathbf{R}, t)$ over a distance $\mathbf{R}$, where

$$
\mathbf{V}(\mathbf{R}, t)=\mathbf{v}(\mathbf{r}(t))-\mathbf{v}(\mathbf{r}(t)+\mathbf{R}) .
$$

With the HS scheme, small seed particles in the fluid scatter light and follow the local flow. A photodetector records the scattered light intensity, which fluctuates due to the motion of the flowing particles. Therefore, the output of the detector is modulated at frequencies equal to the differences in Doppler shifts of all particle pairs in the scattering volume. For each particle pair separated by a distance $R$, this difference is $q \cdot V(R, t)$, and the scattering vector $\mathbf{q}$ has the amplitude $q=(4 \pi n / \lambda) \sin (\theta / 2)$. Here $\theta$ is the scattering angle, $n$ is 
the refractive index of the fluid, and $\lambda$ is the wavelength of the incident light.

With the so-called homodyne method, one measures the intensity autocorrelation function [10]

$$
g(t)=\left\langle I\left(t^{\prime}+t\right) I\left(t^{\prime}\right)\right\rangle=\langle I\rangle^{2}[1+b G(q t, L)],
$$

where $I(t)$ is the intensity of the scattered light and the angle brackets represent a time average over $t^{\prime}$. In the last equality, $L$ is the size of the scattering volume viewed by the photodetector and $b$ is a constant which is chosen so that $G(t=0)=1$. The function $G(q t, L)$ yields information about the velocity differences in the $q$ direction and at various scales $R$ up to $L$. Therefore, one can probe the anisotropy of a turbulent flow by changing the direction of the scattering vector $\mathbf{q}$. Velocity differences at various length scales can be measured by varying the size of the scattering volume. The HS technique measures fluctuations of velocity differences without introducing an invasive probe. Moreover, it is not necessary to invoke Taylor's "frozen-turbulence" assumption [13] to interpret the measurements.

Recently, the present authors and their collaborators have used the HS technique to study turbulent flows in a pipe and in a square tunnel at moderate Reynolds numbers $[6,14,15]$. The Reynolds number $R e$ is defined as $\operatorname{Re}=U l / v$, where $U$ is a characteristic velocity of the flow, $l$ is a characteristic length scale, and $v$ is the kinematic viscosity of the fluid. It was found $[6,14,15]$ that when $\mathrm{Re}$ becomes larger than a transition Reynolds number $\operatorname{Re}_{c}$, the correlation function $G(q t, L)$ has the scaling form

$$
G(q t, L)=G(\kappa),
$$

where $\kappa=q t u(L)$, with $u(L) \sim L^{\xi}$ being the characteristic turbulent velocity at the length scale $L$. The scaling behavior of $G(\kappa)$ indicates [6] that the velocity-density function $P(V, R)$ has the form as shown in Eq. (1). The exponent $\xi$ shows a nontrivial $R$ e dependence and reveals a transitional character when $\mathrm{Re}$ is near $\mathrm{Re}_{c}$. When $\mathrm{Re}$ is below $\operatorname{Re}_{c}$, the characteristic turbulent velocity $u(L)$ is independent of $L \quad(\xi=0)$. In the vicinity of $\operatorname{Re}_{c}$, $\left(\operatorname{Re} \geq \operatorname{Re}_{c}\right), \xi$ increases with $\mathrm{Re}$. At large values of $\mathrm{Re}, \xi$ climbs to, and saturates at, a value close to $\frac{1}{3}$.

The present paper reports a further HS study on the turbulent flow between concentric cylinders in which the inner cylinder rotates. The measurements of $G(q t, L)$ reveal that the turbulence in the cell is neither homogeneous nor isotropic. The predominant turbulent-velocity gradient is in the horizontal plane, whose normal direction is parallel to the rotating axis. The measured correlation function $G(q t, L)$ is found to be of the scaling form as shown in Eq. (3). Put another way, the log-log plots of $G(q t, L)$ at various values of the slit width $L$ and the angular velocity $\omega$, given the scattering geometry and the beam position in the flow cell, can be brought into coincidence by sliding them horizontally with respect to each other. This scaling behavior of $G(q t, L)$ is found to be independent of the scattering geometries and spatial positions. However, when one compares the correlation functions measured in different scattering geometries (keeping the other conditions unchanged) or compares those measured in different spatial positions, it is found that the above scaling description for $G(q t, L)$ is no longer valid.

The paper is organized as follows. Section II describes the experimental apparatus and the methods of data analysis. The results appear in Sec. III, and the work is summarized in Sec. IV.

\section{APPARATUS AND METHODS}

The outer cylinder of the flow cell was made of Plexiglas to admit the incident light and observe the scattering. It was $12.7 \mathrm{~cm}$ both in height and in diameter. The inner rotating cylinder was a smooth brass tube, $9.4 \mathrm{~cm}$ in height and $5.7 \mathrm{~cm}$ in diameter. The tube was shafted along its axis and was mounted on the vertical axis of the outer cylinder through bearings. There was a filling stem on the top lid of the cylinder, and air bubbles could leave from the stem. The flow cell was mounted on the center of a square water bath, which was fixed on an optical table. The water bath was used to index match the cylindrical surface of the flow cell for the scattering measurements. The flow cell was filled with water seeded with small polystyrene latex spheres of diameter $0.1 \mu \mathrm{m}$. These particles follow the local flow [16] and scatter light. The volume fraction of the seed particles was $\sim 10^{-4}$. At this particle concentration, the particle mean spacing is much larger than their diameter (dilute solution) but much smaller than the smallest turbulent scale (sufficient sampling).

Measurements of the correlation function $g(t)$ were performed with a standard light-scattering apparatus and a multichannel correlator (Brookhaven Instruments Model No. BI-2030AT). Figure 1(a) shows the experimental setup. The lens $L_{1}$ focused the incident beam from a 1-W argon-ion laser (Coherent Model No. INNOVA-90, $\lambda=515 \mathrm{~nm}$ ) to form a quasi-onedimensional scattering volume in the flow cell. A typical beam diameter was $0.1 \mathrm{~mm}$. The lens $\mathrm{L}_{2}$ imaged the laser beam without magnification onto a slit $(S)$ of variable width $L$ from 0.1 to $3 \mathrm{~mm}$. Light passing through this slit fell on the photomultiplier (PM), which recorded the time-varying intensity $I(t)$. The photomultiplier was located far behind the slit, so that light was collected from roughly one coherence area. The transistor-transistorlogic pulse train from the photomultiplier went to the correlator, whose output gives the homodyne autocorrelation function $g(t)$ as shown in Eq. (2). Measurements were made at room temperature at the scattering angle $\theta=90^{\circ}$.

The scattering geometries used in the experiment are sketched in Fig. 1(b). The direction of the incident beam was varied in three directions: the azimuthal $(\phi)$ direction [A in Fig. 1(b) ], the radial $(r)$ direction [R in Fig. $1(b)$, and the vertical $(z)$ direction [V in Fig. 1(b)]. For the three scattering geometries, the corresponding scattering vectors $\mathbf{q}=\mathbf{q}_{i}-\mathbf{q}_{s}$ are

$$
\begin{aligned}
& \mathbf{q}_{A}=q_{\phi} \mathbf{e}_{\phi}+q_{r} \mathbf{e}_{r}, \\
& \mathbf{q}_{R}=q_{\phi} \mathbf{e}_{\phi}+q_{r} \mathbf{e}_{r}, \\
& \mathbf{q}_{V}=q_{z} \mathbf{e}_{z}+q_{\phi} \mathbf{e}_{\phi} .
\end{aligned}
$$


One can probe different components of the velocity difference $\mathbf{V}(\mathbf{R}, \mathrm{t})$ by varying the direction of $\mathbf{q}$, since the correlation function $g(t)$ is only sensitive to the product $\mathbf{q} \cdot \mathbf{V}(\mathbf{R}, \mathbf{t})$ [see Eq. (5) below]. Figure 1(b) lists the components of the velocity gradients measured in each geometry. Notice that $L$ is the length of the laser beam viewed by the photomultiplier, and it can be varied by opening the jaws of the slit.

As mentioned in Sec. I, the correlation function $G(q t, L)$ senses the velocity difference $\mathbf{V}(R, t)$ of a pair of points in the turbulent fluid separated by a distance $R$. In the real experiment the photodector receives light from particle pairs having a range of separations $(0<R \leq L)$, and their contributions to the scattering intensity $I(t)$ are proportional to the number fraction of the particle pairs in the scattering volume. When the particles are evenly distributed in a one-dimensional scattering volume of length $L$, the number fraction of particle pairs separated by $R$ is [6] $h(R)=2(1-R / L) / L$. To better assure that the equation is applicable, the incoming laser beam was mildly focused by the lens $L_{1}$ [see Fig. 1(a)]. Because of the frequency beating, the light scattered by each pair of particles contributes a phase factor $\cos (q t V)$ to the intensity correlation function $G(q t, L)$, and $G(q t, L)$ is an incoherent sum of these ensemble-averaged (or timeaveraged) phase factors over all the particle pairs in the scattering volume. Therefore, the function $G(q t, L)$ in Eq. (2) can be written as $[6,8]$

$G(q t, L)=\int_{0}^{L} d R h(R) \int_{-\infty}^{\infty} d V P(V, R) \cos (q t V)$,

where $P(V, R)$ is the velocity-density function and

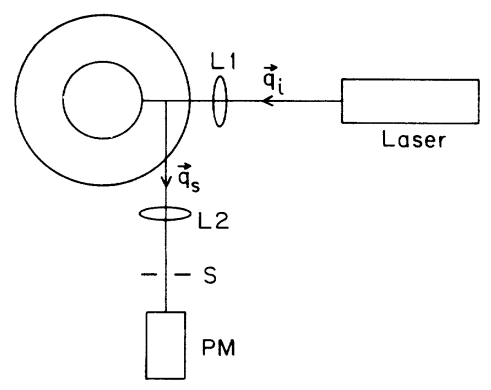

(a)

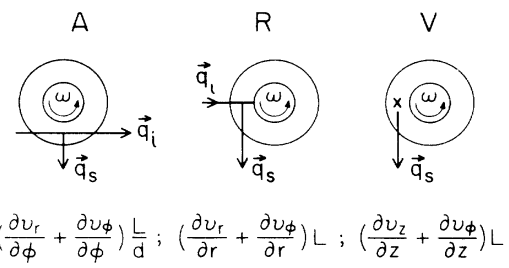

(b)

FIG. 1. Schematic diagram of (a) the experimental setup: $\mathrm{L}_{1}$, $\mathrm{L}_{2}$, lenses; S, adjustable slit; PM, photomultiplier; and (b) the top view of the flow cell and the scattering geometries ( $z$ axis is perpendicular to the paper); $\mathbf{q}_{l}$, incident wave vector; $\mathbf{q}_{s}$, scattering wave vector; $\times$, incident position of the vertical laser beam. The components of velocity difference probed in each geometry are also listed, where $d$ is the distance between the laser beam and the rotation axis, and $L$ is the length of the laser beam viewed by a photodetector.
$V(R, t)$ is the component of $\mathbf{V}(R, t)$ along the scattering vector $\mathbf{q}$.

The function $G(q t, L)$ yields information about the velocity differences in the direction of the scattering vector q, and, senses fluid motions from the largest scale $L$, which is controlled by a slit, down to the smallest scale present in the flow. If the velocity-density function $P(V, R)$ is assumed to be of the scaling form as shown in Eq. (1), Eq. (5) becomes [6]

$$
G(q t, L)=\int_{0}^{L} d R h(R) F(q t u(R))
$$

where $F(x)$ is the Fourier cosine transform of $Q(V / u(R))$. Since the injection rate of the turbulent energy is finite, $F(q t u(R))$ must be a decaying function [17], and the decay rate of $F(q t u(R))$ is then proportional to $q u(R)$ [6]. Equation (6) thus states that $G(q t, L)$ is a weighted sum of decaying functions, each of them characterizing the fluid motion at the length scale $R$, which is in the range between $L$ and the smallest scale of the turbulent flow. For the sake of argument, let us imagine that there were only two modes in a turbulence flow: one was at the scale $L$ and the other was at the Kolmogorov scale $L_{d}$, which is much smaller than $L$. The function $G(q t, L)$ is then a sum of $F(q t u(L))$ and $F\left(q t u\left(L_{d}\right)\right.$ ) (assuming the weighting factor is unity). At small time $t, F(q t u(L))$ dominates the decay of $G(q t, L)$, while $F\left(q t u\left(L_{d}\right)\right)$ is almost a constant. This is because the decay rate of $F(q t u(R))$ is proportional to $R^{1 / 3}$ according to the Kolmogorov theory [1]. At large time $t$, $F(q t u(L))$ has decayed to zero, and $F\left(q t u\left(L_{d}\right)\right)$ determines the decay of $G(q t, L)$.

Therefore, the initial decay of $G(q t, L)$ is dominated by the large-scale motions, and the small-scale motions contribute to the long-time tail of $G(q t, L)$. In principle, one can obtain information about velocity fluctuations at all length scales $(0<R \leq L)$ from a single measurement of $G(q t, L)$ (or by Fourier-transforming $G(q t, L)$ to get the whole frequency spectrum of velocity fluctuations). However, such an analysis requires a detailed modeling of the velocity-density function $P(V, R)$ in Eq. (5). The initial decay time $\tau(L)$ of $G(q t, L)$, on the other hand, can be extracted from the data without modeling the density function $P(V, R)$. As mentioned above, the decay time $\tau(L)$ is proportional to $[q u(L)]^{-1}$, where $u(L)$ is the characteristic velocity difference at the scale $L$. Turbulent motions at various length scales can be examined by measuring the $L$ dependence of the decay time $\tau(L)$.

It is often difficult to determine the initial decay time of a nonexponential decaying function. We therefore use the zeroth moment of $G(q t, L)$ as a definition of the decay time $\tau(L)$. With this definition, which emphasizes the initial decay of $G(q t, L), \tau(L)$ is

$$
\tau(L)=\int_{0}^{\infty} d t G(q t, L)
$$

Using Eq. (7), one can numerically calculate $\tau(L)$ from the measured $G(q t, L)$ without knowing the exact analytic form of $G(q t, L)$. The advantages of using the HS technique are its high spatial resolution and ease of use. 
The high signal-to-noise ratio of the technique ensures that experimental errors are essentially statistical. The uncertainties for $\tau(L)$ in our measurements were below 5\%. At a moderately high scattering intensity ( $I \simeq 10^{5}-10^{6}$ counts $\left./ \mathrm{s}\right)$, it only took $\approx 5 \mathrm{~min}$ to collect the data with an adequate signal-to-noise ratio when the slit width $L=2 \mathrm{~mm}$. At the smallest slit width $L=0.1 \mathrm{~mm}$, it was necessary to collect data for roughly $30 \mathrm{~min}$.

It should be noted that in obtaining Eq. (5) we have assumed [6] that the photodetector is an ideal one (i.e., its detecting area is infinitesimally small) and that $G(q t, L)$ varies on only one dominant time scale $\tau(L) \sim 1 / q u(L)$. In the real experiment, however, $G(q t, L)$ will decay by virtue of the Brownian motion (diffusion) of the seed particles even in the absence of the flow. The corresponding decay time $T_{d}=1 /\left(q^{2} D\right) \approx 10^{-4}$ s where the diffusivity $D$ is inversely proportional to the radius of the diffusers. The time $T_{d}$ was much longer than the decay time $\tau(L)$ associated with turbulent-velocity fluctuations and can be safely ignored in our experiment. Another characteristic decay time is the particle transit time $T_{u}=\sigma / U$, where $\sigma$ is the diameter of the laser beam and $U$ is the mean velocity of the flow. This lifetime-broadening effect was very small in our experiment, the measured decay time $\tau(L)$ being always a factor of 10 shorter than $T_{u}$. The particle transit time imposes a limitation on the smallest length scale $(L \geq \sigma)$ that one can get to in measuring $G(q t, L)$. In our experiment a typical beam diameter was $0.1 \mathrm{~mm}$. The largest length scale $L$ that one can reach in the experiment is determined by the requirement that the light received by the photomultiplier be spatially coherent [10]. This condition was satisfied in our experimental setup as long as the slit width $L$ was less than $2 \mathrm{~mm}$. If the slit opens too widely, the light received by the photomultiplier is no longer spatially coherent and the measured $G(q t, L)$ tends to be flattened at large times [18].

\section{EXPERIMENTAL RESULTS}

In order to map out the turbulent-velocity field, several hundreds of correlation functions have been measured in three different scattering geometries. The function $G(q t, L)$ was measured as a function of the angular velocity $\omega$ of the inner rotating cylinder $(\omega=2 \pi f$, with $f$ in turns per second), the slit width $L$, and the spatial position of the laser beam in the flow cell. The angular velocity $\omega$ was varied from 42 to $385 \mathrm{sec}^{-1}$. The corresponding Reynolds number $\operatorname{Re}=\omega a^{2} / v$ is in the range from $3 \times 10^{4}$ to $3 \times 10^{5}$, where $a(=2.8 \mathrm{~cm})$ is the radius of the inner rotating cylinder and $v$ is the kinematic viscosity of water. Our visual observations show that the fluid in the flow cell is turbulent even at the lowest angular velocity $\omega=42 \mathrm{sec}^{-1}$. The visual observations were made by adding polymeric flakes (Kalliroscope AQ 1000, 1\% concentration) to water. The Reynolds number corresponding to this angular velocity $\left(\operatorname{Re} \simeq 10^{4}\right)$ is much larger than the critical Reynolds number $\operatorname{Re}_{c}$ for the Taylor vortex instability [19] $\left(\operatorname{Re}_{c} \simeq 10^{2}\right)$.

The measurements of $G(q t, L)$ reveal that in our working range of $\omega$, the predominant turbulent-velocity gradient is in the horizontal $(r, \phi)$ plane. Figure 2 compares

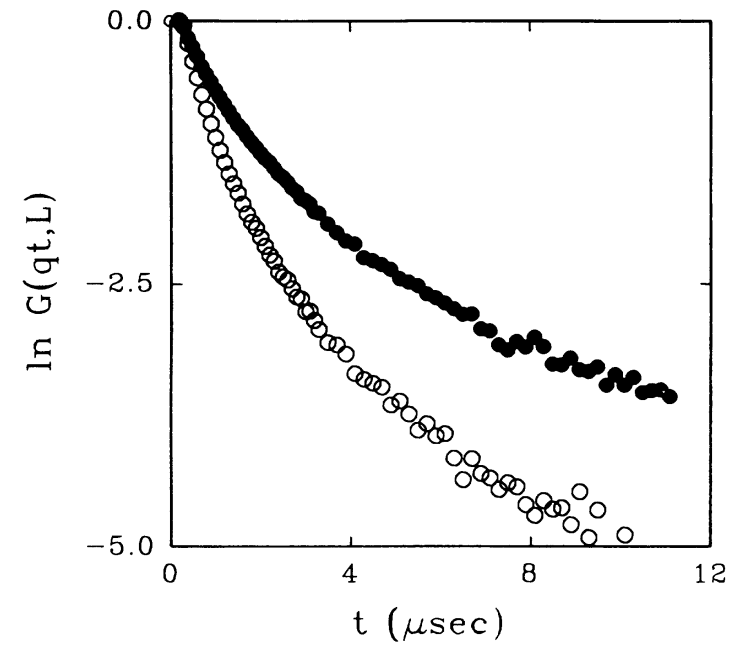

FIG. 2. The measured $G(q t, L)$ in the R geometry (open circles) and in the $\mathrm{V}$ geometry (solid circles). The measurements were made when $\omega=209 \mathrm{sec}^{-1}$ and $L=1.0 \mathrm{~mm}$.

the measured correlation functions $G(q t, L)$ in the $\mathrm{R}$ geometry (open circles) and in the $\mathrm{V}$ geometry (solid circles). The measurements were made when $\omega=209 \mathrm{sec}^{-1}$ and $L=1.0 \mathrm{~mm}$. It is clearly seen that $G(q t, L)$ in the $\mathrm{R}$ geometry decays much faster than that in $\mathrm{V}$, indicating a larger velocity gradient in the $\mathbf{R}$ geometry. The decay time $\tau$ in the $\mathbf{R}$ geometry is $1 \mu \mathrm{sec}$, whereas $\tau=1.8 \mu \mathrm{sec}$ in the $\mathrm{V}$ geometry. The decay time in the $\mathrm{R}$ geometry corresponds to a velocity difference of $4.4 \mathrm{~cm} / \mathrm{sec}$ at the scale of $1 \mathrm{~mm}$. Notice that the measured $G(q t, L)$ in our flow cell is highly nonexponential, which is of different functional form from those measured in a pipe and in a square tunnel $[6,14,15]$.

When the laser beam enters the cell vertically ( $V$ geometry), the measured $G(q t, L)$ is less sensitive to the change of $L$ than to the beam diameter. The decay time $\tau$ increased by a factor of 1.8 when the beam diameter was decreased from 0.2 to $0.1 \mathrm{~mm}$ while only a factor-of-1.15 increase was found in $\tau$ when the slit width $L$ was changed from 1.0 to $0.5 \mathrm{~mm}$. This suggests that the velocity difference over the beam diameter $(\approx 0.1 \mathrm{~mm})$ is larger than that over a vertical distance $L(\approx 1.0 \mathrm{~mm})$. In the horizontal plane the measured $\tau$ for the A geometry is approximately twice larger than that for the $\mathbf{R}$ geometry, which indicates that the strongest velocity gradient in the horizontal plane is in the radial direction. Therefore, the turbulence in our flow cell is neither homogeneous nor isotropic. The differences of the velocity field in different spatial positions and in different orientations are also shown in its scaling properties, to be discussed below.

In spite of this anisotropy, it is found that the measured $G(q t, L)$ has the scaling form as shown in Eq.(3). Log-log plots of $G(q t, L)$ for various values of the slit width $L$ and the angular velocity $\omega$, at the fixed scattering geometry and the fixed beam position in the flow cell, can be brought into coincidence by sliding them horizontally with respect to each other. The decay time $\tau(L)$ defined in Eq. (7) [see the discussion about $\tau(L)$ below] quantitatively characterizes the amount of the horizontal transla- 
tion that is required to bring the correlation functions into coincidence. Figure 3 shows typical $G(\kappa)$ as a function of $\kappa=t / \tau(L)$ for various values of $\omega$ and $L$. Since $\tau(L) \simeq[q u(L)]^{-1}$, where $u(L)$ is the characteristic velocity difference at the scale $L$, the scaling argument $\kappa$ can also be written as $\kappa=q t u(L)$. The correlation functions in Fig. 3(a) were measured at the $R$ geometry and those in Fig. 3(b) were obtained at the $V$ geometry. Similar scaling behavior for the measured $G(q t, L)$ is found at the A geometry and in other beam positions.

As mentioned in Sec. I, a scaling $G(\kappa)$ can be obtained if the velocity-density function $P(V, R)$ in Eq. (5) has the scaling form as shown in Eq. (1) [6]. This scaling form for $P(V, R)$ implies that the turbulent velocity $V(R, t)$ is scale invariant, i.e., all its moments $\left\langle V(R, t)^{n}\right\rangle$ obey a power law of $R$ [6]. This is expected from the Kolmogorov theory of isotropic turbulence [1-3]. Recently, Knight and Sirovich [7] showed that the above scaling arguments for homogeneous and isotropic turbulence can
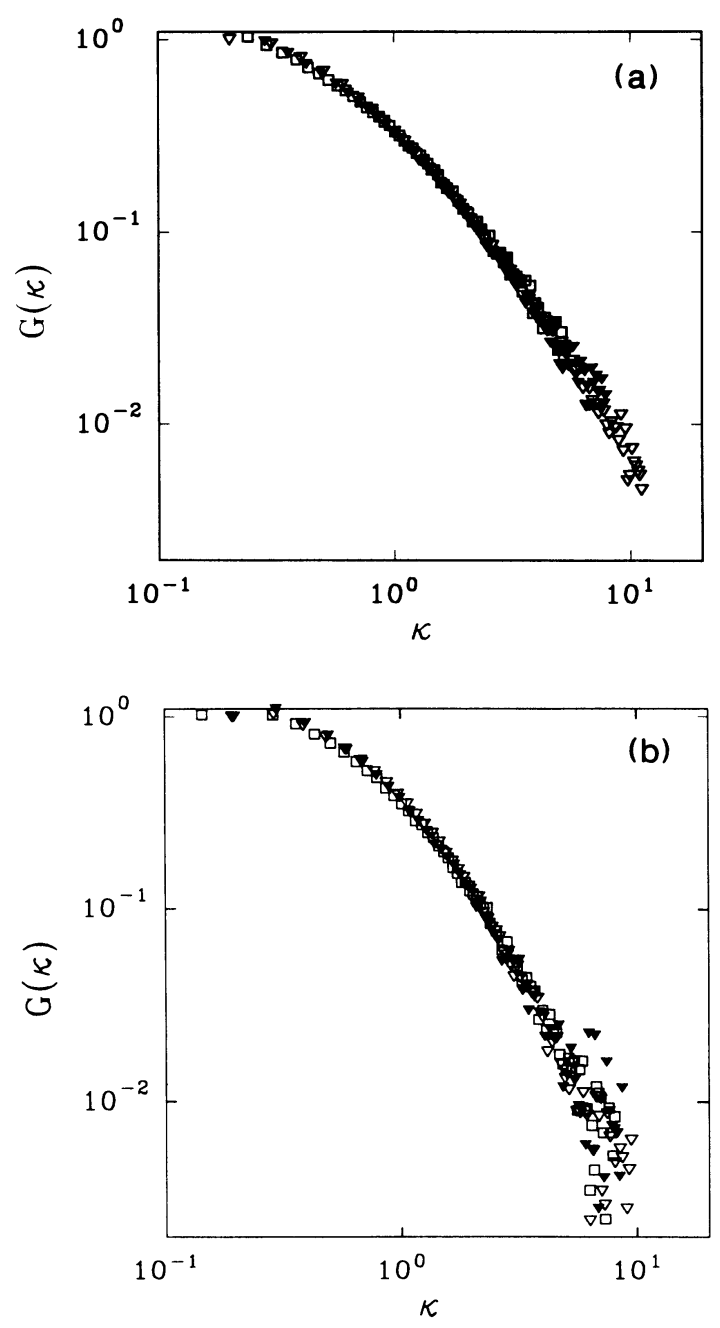

FIG. 3. The scaling function $G(\kappa)$ vs $\kappa=q t u(L) \simeq t / \tau(L)$ : (a) in the $\mathbf{R}$ geometry and (b) in the $\mathbf{V}$ geometry. The experimental conditions are $L=1.0 \mathrm{~mm}, \omega=209 \mathrm{sec}^{-1}$ (open triangles); $L=0.3 \mathrm{~mm}, \omega=262 \mathrm{sec}^{-1}$ (solid triangles); and $L=0.2$ $\mathrm{mm}, \omega=209 \mathrm{sec}^{-1}$ (open squares). be extended to certain turbulent flows lacking homogeneity and isotropy, and suggested this might work more broadly. Our turbulent flow is certainly neither isotropic nor homogeneous, and the scaling results in Fig. 3 support the scaling arguments by Knight and Sirovich.

Another important quantity in the statistical description of turbulence is the scaling velocity $u(R)$ as a function of the spatial separation $R$. The scale dependence of the scaling velocity can be examined by measuring the $L$ dependence of the decay time $\tau(L)$, since $\tau(L) \sim[q u(L)]^{-1}$. It is found that the decay time $\tau(L)$ as a function of $L$ obeys a power law, $\tau(L) \sim L^{-\xi}$ [i.e., $\left.u(L) \sim L^{\xi}\right]$. Figure 4 shows the measured $\tau(L)$ as a function of $L$ at the fixed $\omega=209 \mathrm{sec}^{-1}$. The two curves in Fig. 4(a) were obtained in the $V$ geometry when the vertical laser beam was $1.2 \mathrm{~cm}$ away from the outer wall of the flow cell (upper curve) and when the beam was $2.5 \mathrm{~cm}$ away from the wall (lower curve). Figure 4(b) compares the measured $\tau(L)$ in the $\mathrm{V}$ geometry (upper curve) with that in the $\mathbf{R}$ geometry (lower curve).

It is seen from Fig. 4 that $\log [\tau(L)]$ lies on a straight
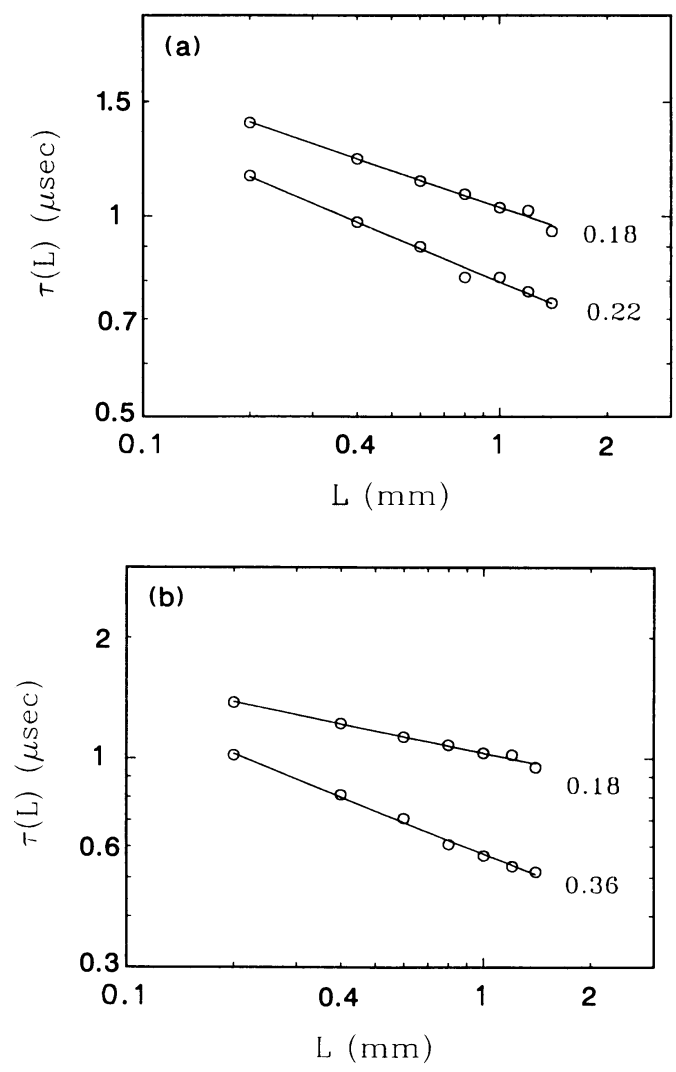

FIG. 4. Variations of the decay time $\tau(L)$ with the slit width $L$ measured at $\omega=209 \mathrm{sec}^{-1}$. The solid lines are power-law fits, and the number labeled beside a line is the slope of that line. (a) shows the measurements in the $\mathrm{V}$ geometry. The upper curve was obtained when the incident laser beam is $1.2 \mathrm{~cm}$ away from the outer wall of the flow cell; the lower curve was obtained when the beam is $2.5 \mathrm{~cm}$ away from the wall. (b) compares the measurement in the $\mathrm{V}$ geometry (upper curve) with that in the $\mathbf{R}$ geometry (lower curve). 
line when $L$ is in the range $0.2 \mathrm{~mm} \leq L \leq 1.4 \mathrm{~mm}$. The largest value of $L$ over which scaling is observed was limited by the coherent area viewed by the photomultiplier, as discussed at the end of Sec. II. The lower cutoff at small $L$ was controlled by the laser-beam diameter $(\approx 0.1$ $\mathrm{mm}$ ). From the straight-line segment (solid lines in Fig.4), we obtain the slope $\xi$, which has different values for different scattering geometries and different beam positions. Table I lists the typical values of the exponent $\xi$ measured for the three scattering geometries when $\omega=209 \mathrm{sec}^{-1}$. The experimental uncertainties for the slope $\xi$ are below $10 \%$.

For a laminar flow one expects that the exponent $\xi=1[u(L) \sim L]$ in the $\mathbf{R}$ geometry and that $\xi=0$ in the geometries $\mathbf{A}$ and $\mathrm{V}$. For fully developed turbulence, the exponent $\xi=\frac{1}{3}$ in all geometries according to the Kolmogorov theory $[1]\left[u(L) \sim L^{1 / 3}\right]$. As mentioned in Sec. I, early HS measurements for turbulent flows in a pipe and in a square tunnel $[6,14,15]$ have shown that the $\operatorname{Re}$ dependence of the exponent $\xi$ reveals a transitional character when $R e$ is near the transition Reynolds number $\operatorname{Re}_{c}$. When $R e$ is above $\operatorname{Re}_{c}, \xi$ increases with $R e$ and saturates at a value close to $\frac{1}{3}$ at large values of Re. We, therefore, expect that for the turbulent flow in the concentric cell, $\xi$ will approach $\frac{1}{3}$ from above in the $\mathbf{R}$ geometry when $R e$ is increased. This is because there is a laminar shear in the $\mathrm{R}$ geometry. It is also expected that the exponent $\xi$ will approach $\frac{1}{3}$ from below in the $A$ and $\mathrm{V}$ geometries. In fact, it was found that the value of $\xi$ in the $\mathrm{V}$ geometry was increased from 0.19 to 0.28 when the angular velocity $\omega$ was changed from 136 to $262 \mathrm{sec}^{-1}$.

It is clearly seen from Table I that the values of $\xi$ for the geometries $\mathbf{A}$ and $\mathbf{R}$ are close to the Kolmogorov value. This indicates that the turbulence in the two geometries is nearly fully developed. Turbulence in the $\mathrm{V}$ geometry, on the other hand, is weak because the value of $\xi$ is below the one for fully developed turbulence. Therefore, the difference of the exponent $\xi$ in the three geometries, as shown in Fig. 4(b) and Table I, reveals the anisotropy of our turbulent flow. Figure 4(a) shows that the change of the exponent $\xi$ is small when one varies the position of the incident laser beam while keeping the scattering geometry (beam and detector directions) unchanged. However, the absolute value of the decay time $\tau(L)$ is increased $[u(L)$ is reduced] as the laser beam is moved toward the outer wall. This indicates that the flow near the outer wall is less turbulent than that in the middle region, reflecting the inhomogeneity of the turbulence.

The above scaling properties of the correlation function $G(q t, L)$ are observed over various length scales and Reynolds numbers when the spatial position of the laser beam and the scattering geometry are fixed. However, when one compares the correlation functions measured at

TABLE I. The typical values of the exponent $\xi$ measured for the three scattering geometries when $\omega=209 \mathrm{sec}^{-1}$.

\begin{tabular}{lccc}
\hline \hline Geometry & $\mathrm{A}$ & $\mathrm{R}$ & $\mathrm{V}$ \\
Typical $\xi$ & 0.34 & 0.36 & 0.18 \\
\hline \hline
\end{tabular}

different scattering geometries (keeping the other conditions unchanged) or compares those measured in different spatial positions, it is found that these functions do not scale mutually. Figure 5(a) compares the measured $G(\kappa)$ at three scattering geometries. The two correlation functions measured in the $\mathbf{A}$ and $\mathbf{R}$ geometries deviate from each other at small times, and can be superimposed at large times. This can be explained by the fact that the large-scale motions are not isotropic, and these effects from boundaries are much reduced as turbulence cascades down to smaller scales. Notice that the turbulence in the two scattering geometries is nearly fully developed, and the values of their scaling exponents $\xi$ are close to the Kolmogorov value (see Table I). In the above analysis we have used the fact that the initial decay of
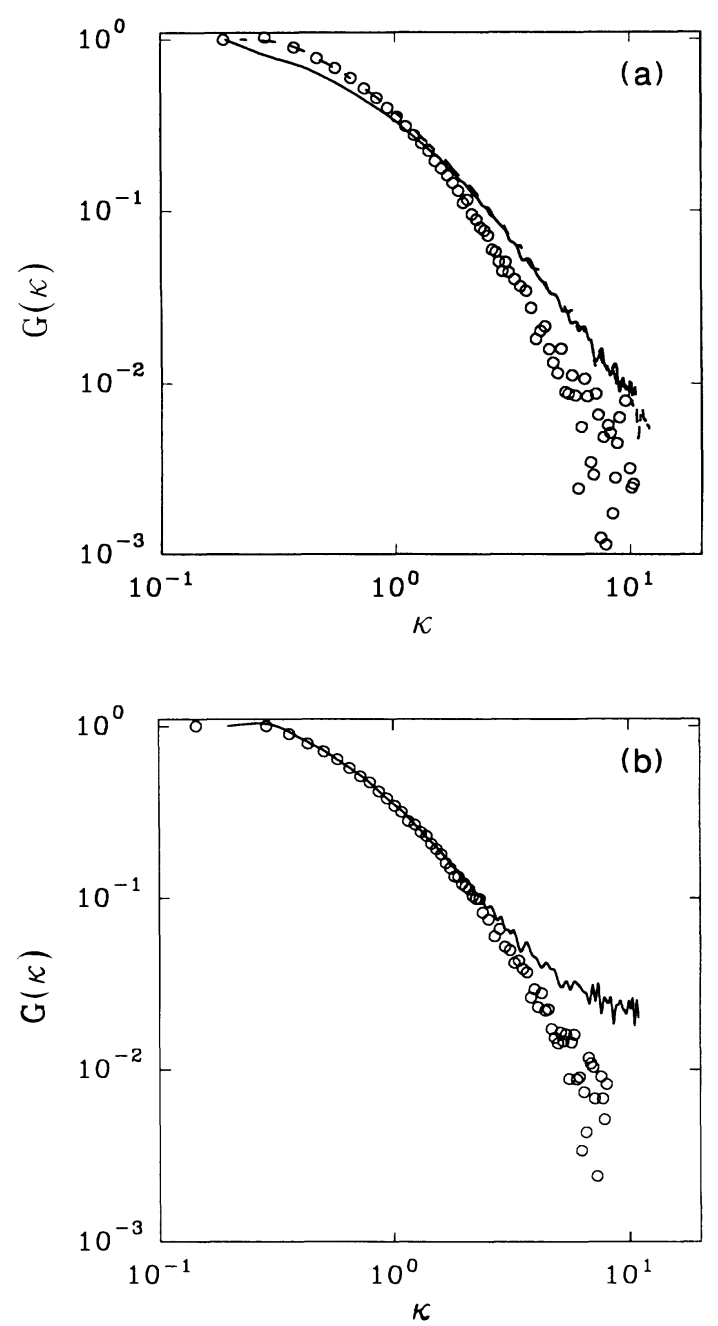

FIG. 5. The scaling function $G(\kappa)$ vs $\kappa=q t u(L) \simeq t / \tau(L)$. (a) shows the measurements performed when $\omega=209 \mathrm{sec}^{-1}$, $L=1.0 \mathrm{~mm}$, and in various scattering geometries: A geometry (solid curve), R geometry (dashed curve), and V geometry (open circles). (b) shows the measurements in the $\mathrm{V}$ geometry when $\omega=209 \mathrm{sec}^{-1}$ and $L=0.2 \mathrm{~mm}$. The open circles were obtained when the incident laser beam is $1.2 \mathrm{~cm}$ away from the outer wall of the flow cell; the solid curve was obtained when the beam is $2.5 \mathrm{~cm}$ away from the wall. 
$G(q t, L)$ is dominated by the large-scale motions, and the small-scale motions only contribute to the long-time tail of $G(q t, L)$. [See Sec.II for the discussion about how the function $G(q t, L)$ senses fluid motions from the largest scale $L$ down to the smallest one.] For the V geometry the measured $G(q t, L)$ fails to scale with those measured in geometries A and R. The fluid flow in this geometry is less turbulent, as indicated by a slow decay of $G(q t, L)$ and a small exponent $\xi$. Figure $5(\mathrm{a})$ is suggestive that small-scale velocity fluctuations become isotropic when the scaling exponent $\xi$ approaches its asymptotic value.

Figure 5(b) compares $G(\kappa)$ measured in two different spatial positions when the laser beam enters the cell vertically (V geometry) with $\omega=209 \mathrm{sec}^{-1}$ and $L=0.2 \mathrm{~mm}$. The open circles were obtained when the incident laser beam is $1.2 \mathrm{~cm}$ away from the outer wall of the flow cell; the solid curve was obtained when the beam is $2.5 \mathrm{~cm}$ away from the wall. It can be seen from Fig. 5(b) that the two correlation functions fail to scale with each other at large times, suggesting that the small-scale motions in the two spatial positions are very different. This can be understood by the fact that the flow near the outer wall is less turbulent than that in the middle region [see the discussion about Fig. 4(a)]. Figures 5(a) and 5(b) indicate that the functional form of $G(q t, L)$, and hence the velocity-density function $P(V, R)$ [see Eq. (5)], changes when the scattering geometry and/or the spatial position are changed.

Our results in this section, therefore, suggest that the scaling argument can still be used to describe inhomogeneous turbulent flows and that the exact functional form of the velocity-density function $P(V, R)$ (which is of scaling form) may vary with spatial positions and orientations, reflecting the inhomogeneity and anisotropy of the turbulence. To further verify the conclusion, we deliberately inserted four radial baffle plates placed symmetrically on the wall of the outer cylinder. These baffle plates introduced additional inhomogeneity and anisotropy into the flow. The width of each baffle place was 1.3 $\mathrm{cm}$, and its height was the same as that of the outer wall. This is a typical design for a mixing vessel [20].

In the baffled cell one can clearly see that in the $z$ direction there are large-scale velocity fluctuations, which are absent in the unbaffled cell. When the fluid hits the corner where the outer wall and a baffle plate meet, its flow direction is forced to change either in the horizontal plane (where the fluid element has to be stretched) or in the vertical direction (up-down motion). The vertical motion was observed by eye through the addition of polymeric flakes into water. The measured $G(q t, L)$ shows that in the baffled cell the velocity gradient in the $r$ direction is reduced. The maximum reduction of the velocity gradient occurs at small angular velocities $\left(\omega \simeq 94 \mathrm{sec}^{-1}\right)$; at large angular velocities $\left(\omega \geq 203 \mathrm{sec}^{-1}\right)$ the reduction becomes smaller. In the $\phi$ direction the velocity gradient is approximately the same as in the unbaffled cell. Thus the insertion of the baffle plates enhances velocity fluctuations in the $z$ direction and reduces the velocity gradient in the $r$ direction. One may view the turbulence in the baffled cell as a turbulent wake generated by the baffle plates. Furthermore, this turbulent wake is steady and
TABLE II. The typical values of the exponent $\xi$ measured for the three scattering geometries in the baffled cell when $\omega=209 \mathrm{sec}^{-1}$.

\begin{tabular}{lccc}
\hline \hline Geometry & $\mathrm{A}$ & $\mathrm{R}$ & $\mathrm{V}$ \\
Typical $\xi$ & 0.3 & 0.45 & 0.21 \\
\hline \hline
\end{tabular}

spatially extends to the bulk region of the gap between the two cylinders.

We now compare the scaling results in the unbaffled cell with those in the baffled cell. Similar to the situation in the unbaffled cell, the measured $G(q t, L)$ in the baffled cell is also found to be of the scaling form as shown in Eq. (3). Log-log plots of $G(q t, L)$ for various values of $L$ and $\omega$, given the scattering geometry and the beam position in the flow cell, can be superimposed by scaling the time axis to $\kappa=t / \tau(L)$. Figure 6 shows the typical $G(\kappa)$ as a function of $\kappa=q t u(L) \simeq t / \tau(L)$ for various values of $\omega$ and $L$. These correlation functions were measured in the baffled cell at the A geometry. The scaling behavior of the measured $G(q t, L)$ is also found in other scattering geometries.

The decay time $\tau(L)$ in the baffled cell still obeys the power law $\tau(L) \sim L^{-\xi}$. Table II lists the typical values of the exponent $\xi$ measured for the three scattering geometries in the baffled cell when $\omega=209 \mathrm{sec}^{-1}$. The anisotropy of the flow in the baffled cell is clearly shown by the difference of the exponent $\xi$ in the three geometries. It is seen from Table II that the value of $\xi$ for the A geometry is close to the Kolmogorov value, indicating that the turbulence in this geometry is nearly fully developed. Turbulence in the $\mathrm{V}$ geometry, on the other hand, is weak because the value of $\xi$ is below the one for fully developed turbulence. By comparing Table II with Table I, we find that the turbulence in the baffled cell at the $\mathrm{R}$ geometry is reduced. The measured $\xi$ is far away from the Kolmogorov value. In fact, it is found

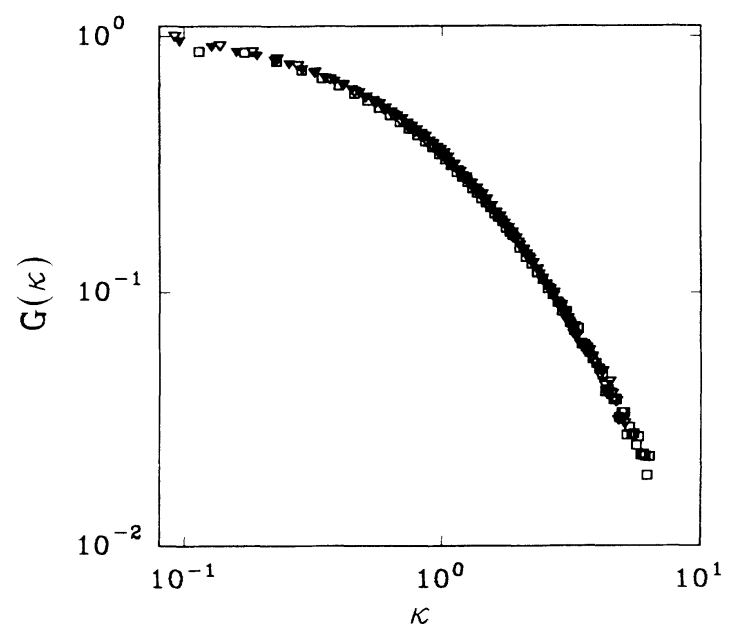

FIG. 6. The scaling function $G(\kappa)$ vs $\kappa=q t u(L) \simeq t / \tau(L)$ in the baffled cell at the A geometry. The experimental conditions are $L=1.0 \mathrm{~mm}, \omega=209 \mathrm{sec}^{-1}$ (open triangles); $L=0.2 \mathrm{~mm}$, $\omega=209 \mathrm{sec}^{-1}$ (solid triangles); and $L=0.5 \mathrm{~mm}, \omega=262 \mathrm{sec}^{-1}$ (open squares). 


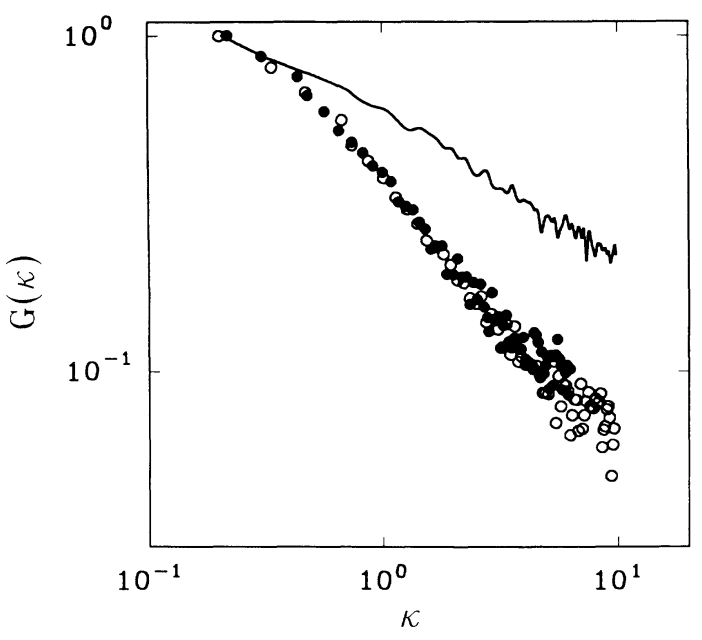

FIG. 7. The scaling function $G(\kappa)$ vs $\kappa=q t u(L) \simeq t / \tau(L)$ in the baffled cell at the $\mathrm{R}$ geometry. The correlation functions were measured when $\omega=209 \mathrm{sec}^{-1}$ and at various slit widths: $L=1.3 \mathrm{~mm}$ (open circles), $L=0.6 \mathrm{~mm}$ (solid circles), and $L=0.2 \mathrm{~mm}$ (solid curve).

that the measured $G(q t, L)$ is a scaling function $G(\kappa)$ only when the slit width $L \geq 0.6 \mathrm{~mm}$ in this geometry. Figure 7 shows the correlation functions $G(\kappa)$ measured in the baffled cell at various values of $L$. These functions were measured when $\omega=209 \mathrm{sec}^{-1}$ and at the $\mathrm{R}$ geometry. It can be seen that the correlation function $G(q t, L)$ at $L=0.2 \mathrm{~mm}$ fails to scale with the other $G(q t, L)(L \geq 0.6 \mathrm{~mm})$. This is because the turbulence in this geometry is very weak at small scales, so that $G(q t, L)$ changes its functional form at small length scales.

The above results suggest that scaling concepts can also be used to describe the turbulence in the baffled cell. However, when one compares the correlation function measured in the baffled cell with that in the unbaffled cell (keeping the other conditions unchanged), it is found that the above scaling description for $G(q t, L)$ is no longer valid. Figure 8 compares the measured $G(\kappa)$ in the unbaffled cell (solid curve) and in the baffled cell (open circles). The two correlation functions were measured when $L=0.6 \mathrm{~mm}, \omega=209 \mathrm{sec}^{-1}$, and in the $\mathrm{V}$ geometry. From Fig. 8 we conclude that the turbulent flows between the two flow cells in the $\mathrm{V}$ geometry are very different, which yields a different functional form for $G(q t, L)$ and hence for the density function $P(V, R)$.

\section{CONCLUSION}

The technique of photon-correlation homodyne spectroscopy was used to measure turbulent-velocity differences in a Couette cell in which the inner cylinder rotates. Different components of the velocity differences were probed by changing the direction of the scattering vector q. Velocity differences at various length scales were measured by varying the size of the scattering

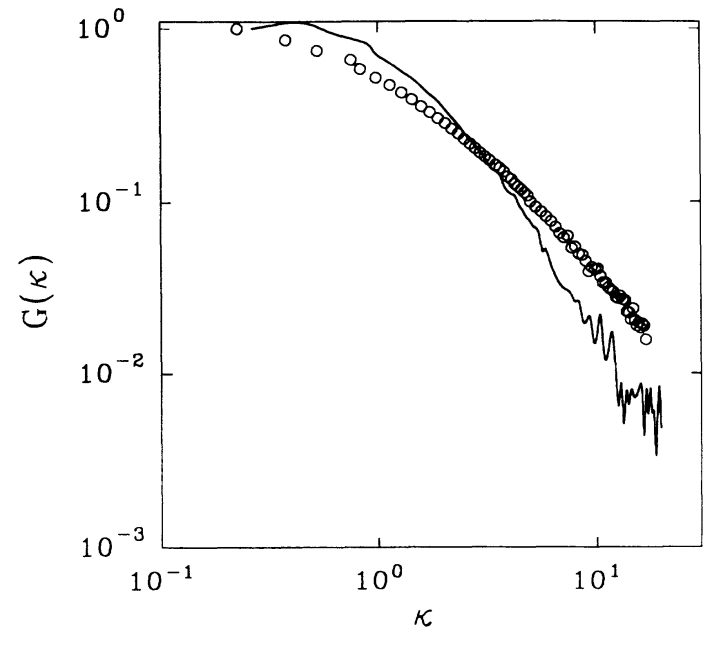

FIG. 8. The scaling function $G(\kappa)$ vs $\kappa=q t u(L) \simeq t / \tau(L)$ in the baffled cell (open circles) and in the unbaffled cell (solid curve). The two correlation functions were measured when $\omega=209 \mathrm{sec}^{-1}, L=0.6 \mathrm{~mm}$, and in the $\mathrm{V}$ geometry.

volume. The measurements of the correlation function $G(q t, L)$ reveal that the turbulence in the flow cell is neither homogeneous nor isotropic. The predominant turbulent-velocity gradient is in the horizontal plane, whose normal direction is parallel to the rotating axis.

The measured $G(q t, L)$ is found to be of the scaling form $G(q t u(L))$ with $u(L) \sim L^{\xi}$, where $u(L)$ is the characteristic turbulent velocity at the length scale $L$. The log-log plots of $G(q t, L)$ for various values of the slit width $L$ and the angular velocity $\omega$, at the fixed scattering geometry and the fixed beam position in the flow cell, can be brought into coincidence by sliding them horizontally with respect to each other. The scaling behavior of $G(q t, L)$ is found to be independent of the scattering geometries and the flow cells. The value of $\xi$ varies with scattering geometries and with flow cells. A scaling $G(q t u(L))$ indicates that the velocity-density function $P(V, R)$ has a scaling form $Q(V / u(R)) / u(R)$.

However, when one compares the correlation functions measured in different scattering geometries (keeping the other conditions unchanged) or compares those measured in different spatial positions, it is found that the above scaling description for $G(q t, L)$ is no longer valid. Our results, thus, suggest that the scaling argument can still be used to describe anisotropic and inhomogeneous turbulent flows and that the exact functional form of the velocity-density function $P(V, R)$ (which is of scaling form) may vary with spatial positions and orientations in turbulent flows, reflecting the inhomogeneity and anisotropy of the turbulence.

\section{ACKNOWLEDGMENT}

This work was supported in part by the National Science Foundation under Grant No. DMR-8914351. 
[1] A. N. Kolmogorov, C.R. Dokl. Acad. Sci. URSS 30, 301 (1941); 31, 538 (1941).

[2] U. Frisch, in Turbulence and Predictability in Geophysical Fluid Dynamics and Climate Dynamics, edited by M. Ghil, R. Benzi, and G. Parisi (North-Holland, Amsterdam, 1985), p.71.

[3] U. Frisch, P. Sulem, and M. Nelkin, J. Fluid Mech. 87, 719 (1978).

[4] R. H. Kraichnan, J. Fluid Mech. 62, 305 (1974).

[5] C. W. van Atta and J. Park, in Statistical Models and Turbulence, edited by M. Rosenblatt and C. W. van Ata, Lectures Notes in Physics Vol. 12 (Springer, Berlin, 1972) p. 402.

[6] P. Tong, W. I. Goldburg, C. K. Chan, and A. Siriat, Phys. Rev. A 37, 2125 (1988)

[7] B. Knight and L. Sirovich, Phys. Rev. Lett. 65, 1356 (1990).

[8] A. Onuki, Phys. Lett. A 127, 143 (1988).

[9] P. J. Bourke et al., J. Phys. A 3, 216 (1970).

[10] B. J. Berne and R. Pecora, Dynamic Light Scattering (Wiley, New York, 1976).

[11] L. E. Drain, The Laser Doppler Technique (Wiley, New
York, 1980).

[12] F. Durst, A. Melling, and J. H. Whitlaw, Principles and Practice of Laser-Doppler Anemometry, 2nd ed. (Academic, New York, 1981).

[13] See e.g., H. Tennekes and J. L. Lumley, A First Course in Turbulence (MIT Press, Cambridge, MA, 1972).

[14] P. Tong and W. I. Goldburg, Phys. Fluids 31, 2841 (1988); Phys. Lett. A 127, 147 (1988); W. I. Goldburg and P. Tong, Phys. Scr. 40, 424 (1989).

[15] W. I. Goldburg, P. Tong, and H. K. Pak, Physica D 38, 134 (1989); H. K. Pak, W. I. Goldburg, and A. Sirivat, Fluid Dynamics Res. 8, 19 (1991).

[16] See, e.g., L. E. Drain, The Laser Doppler Technique (Ref. [11]), p. 183.

[17] A Onuki, Prog. Theor. Phys. S99, 382 (1989)

[18] W. I. Goldburg (unpublished).

[19] R. C. DiPrama and H. L. Swinney, in Hydrodynamic Instablities and the Transition to Turbulence, edited by H. L. Swinney and J.P. Gollub (Springer, Berlin, 1985).

[20] S. Nagata, Mixing: Principles and Applications (Wiley, New York, 1975). 